\title{
A Eletrostática na Corrente Elétrica em Fios
}

Electrostatic for the Electrical Current in Wires

\author{
G. F. Leal Ferreira e Mariângela T. de Figueiredo \\ Instituto de Física de São Carlos, DFCM \\ CP 369, 13560-970, São Carlos, SP
}

Recebido em 06 janeiro, 2003. Aceito em 08 de setembro, 2003.

\begin{abstract}
O artigo refere-se à questão 'Quem cria o campo elétrico que move as cargas na corrente elétrica em fios?'. Através de modelo simples, mostra-se que uma distribuição de cargas, variando linearmente ao longo do fio e depositada na sua superfície, cria campo uniforme no seu interior. O modelo utilizado é o de um cabo coaxial fechado em ambas as extremidades, com potenciais convenientemente escolhidos em suas paredes. Com este estudo, torna-se possível ilustrar a importante ligação conceitual entre a Eletrostática e a Eletrodinâmica, historicamente tão importante no desenvolvimento do Eletromagnetismo.
\end{abstract}

The article is aimed to answer the question 'Who creates the electric field inside a wire supporting an electric current?'. By means of a simple model it is shown that a surface charge distribution varying linearly along the wire creates an uniform electric field inside it. The utilized model is that of a closed at both ends coaxial cable, whose walls are kept at conveniently chosen potentials. This study allows us to bridge the way from Electrostatics to Electrodynamics, historically so important in the development of the electromagnetic science.

\section{Introdução}

No ensino de eletricidade, o capítulo sobre 'correntes contínuas' desperta pouco interesse porque, praticamente, versa sobre as leis de Kirchoff em circuitos, assunto em que os alunos já têm alguma habilidade. Mas, historicamente, foi muito importante a passagem da Eletrostática à Eletrodinâmica, pois conceitos desenvolvidos na primeira foram usados na descrição da segunda: a corrente elétrica gerada por pilhas elétricas foi identificada como carga em movimento. A pilha, pela sua força eletromotriz, traz a carga circulante de um pólo ao outro no seu interior, e mantém a diferença de potencial entre as extremidades do fio condutor que fecha externamente o circuito. Mas quem cria o campo elétrico necessário para mover a corrente no interior do fio? Não é razoável supor que cargas localizadas nos pólos da pilha possam criar aquele campo uniforme ao longo de todo o fio. Como diz o famoso J. D. Jackson [1], 'The significance of the surface electric charge densities associated with current-carrying circuit is often not appreciated. In general, the conductors of a current-carrying circuit must have nonuniform surface charge densities on them...'. (A importância das cargas superficiais associadas a correntes em circuito é muitas vezes não reconhecida. Em geral, os condutores de um circuito com corrente devem ter distribuição de carga superficial não uniforme). E é um problema de Eletrostática explicar o aparecimento do campo uniforme do interior do fio através de distribuição de cargas superficiais. Dos livrostexto mais antigos só os das referências [2] e [3], do nosso conhecimento, abordam o assunto (ver porém [4]), como co- mentaremos em breve. Mais recentemente, um punhado de artigos [1, 4-10] dedica-se ao assunto e, em especial, o da referência [4] historia o desenvolvimento da relação entre a Eletrostática e a Eletrodinâmica. Brevemente, ela foi percebida por Ampère em 1820 e, em 1827, Ohm, ao estabelecer a lei com seu nome, procurou justificar o aparecimento do campo elétrico, isto é, de um gradiente de potencial no interior do fio, através de um gradiente de carga volumétrica, numa teoria em que as duas espécies de carga eram móveis. Coube a Kirchhoff (1850) mostrar que a corrente uniforme necessitava apenas um gradiente de potencial criado por um gradiente de carga superficial, estabelecendo definitivmente aquela relação. Em especial, na referência [9], o problema de dois cabos infinitos, paralelos, com correntes de sentidos opostos, é tratado e na [10], em alentado estudo, abordase o caso do condutor toroidal com corrente, permitindo a obtenção do limite do fio com corrente. Em [11] reportamse resultados experimentais.

O modelo físico que utilizaremos será uma adaptação daquele apresentado em [2] e [3], em que se trata a situação idealizada de um cabo co-axial infinito, constando de um fio central resistivo, envolto por cilindro condutor perfeito, estendendo-se ao infinito de um e do outro lado da seção em que o potencial é nulo e provendo a corrente de retorno. Mantendo a solução como em [2] e [3], no tratamento da Seção II o cabo co-axial é já considerado semiinfinito, fechando-o numa extremidade por uma tampa de condutor perfeito, ligando o fio central ao cilindro externo. Na Seção III, fecha-se o cabo co-axial numa outra seção 
por uma tampa 'resistiva ', na qual o potencial varia do valor no cilindro envoltório - que é o mesmo da primeira extremidade do fio -, ao potencial em que a outra extremidade do fio resistivo central é mantido. Com isso, em termos de resistências, a segunda tampa e o fio central formam um sistema de duas resistências em paralelo. Isto é, no nosso modelo adicionamos condições de contorno físicas à solução conhecida, de maneira a mantê-la válida para o caso finito. Note-se que, na prática, as resistividades, espessuras e comprimentos devem ser escolhidos de forma a que, aproximadamente, todo o potencial caia ao longo dessa segunda tampa, em comparação à queda na primeira tampa e cilindro envoltório. Continuando, na seção IV calculamos as cargas depositadas nas superfícies condutoras do cabo finito e na seção V, usando [10] fazemos algumas considerações sobre as cargas que se devem depositar em fios finos percorridos por corrente.

Acima empregamos termos como 'condutor perfeito' e 'condutor resistivo'. Na situação estudada aqui, poderíamos definir o cobre como condutor perfeito, e o carvão, cerca de 2000 vezes mais resistivo (ver Seção IV), como condutor resistivo.

\section{Cálculo do campo elétrico}

Seja a Fig.1, adaptada do tratamento em [1] e [2]. O cabo representado tem um condutor interno, cilíndrico, resistivo, de eixo $z$ e raio $a$, envolto, por um cilindro co-axial de condutor perfeito, de raio $b$. A seção toda em $z=0$ está a potencial zero, usando-se para isso disco de condutor perfeito ligando, em $z=0$, a superfície do condutor interno ao externo. O cilindro de raio $b$ também está a potencial nulo. No condutor interno, de condutividade $g$, há uma corrente $I$, movida por campo elétrico uniforme $E$, com

$$
g E \pi a^{2}=I .
$$

Temos, então, as seguintes condições para o potencial $V(r, z)$ :

$$
\begin{gathered}
V(r, z)=-E z, \text { para } z \geq 0, r \leq a \\
\nabla^{2} V(r, z)=0, \text { para } z \geq 0 \text { e } a \leq r \leq b,
\end{gathered}
$$

com

$$
V(a, z)=-E z \text { e } V(b, z)=0 .
$$

À região $a<r<b, z>0$, chamaremos de 'ar'. Como

$$
\nabla^{2} V(r, z)=\frac{1}{r} \frac{\partial}{\partial r}\left(r \frac{\partial V}{\partial r}\right)+\frac{\partial^{2} V}{\partial z^{2}}
$$

vê-se que $V(r, z)=z F(r)$ é solução da Eq.4, obtendo-se para $F(r)$ o resultado

$$
F(r)=K \ln r+C
$$

que, com as condições da Eq.3, fornece para $V(r, z)$

$$
V(r, z)=-z \frac{I}{\pi a^{2} g} \frac{\ln \frac{r}{b}}{\ln \frac{a}{b}} .
$$

As equipotenciais entre $r=a$ e $r=b$ estão esboçadas na Fig.1.

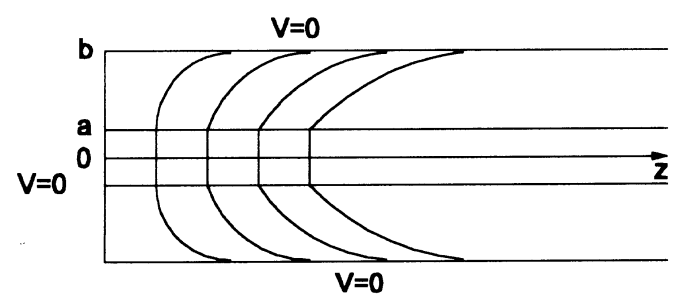

Figura 1. Cabo co-axial semi-infinito, formado do condutor resistivo central ao longo do eixo $z$, de condutividade $g$. O cilindro externo, de raio $b$, é de condutor perfeito, bem como o disco que fecha o cabo em $z=0$, ambas mantidas ao potencial nulo. Potencial negativo é aplicado ao condutor central, em $z \rightarrow \infty$.

\section{Fechando o cabo}

Em [1] e [2], o cabo co-axial é apresentado como se estendendo de $-\infty$ a $+\infty$. Nós, na Fig.1, já o fizemos semiinfinito, começando em $z=0$, fechando-o por meio de placa circular de condutor perfeito ao potencial zero. O potencial negativo está sendo aplicado ao fio central em $z \rightarrow$ $\infty$, uma característica irrealista do modelo, que pretendemos remediar. Notemos que o potencial na seção $z=h$, entre $a$ e $b$, Eq.6, é

$$
V(r, h)=-h \frac{I}{\pi a^{2} g} \frac{\ln \frac{r}{b}}{\ln \frac{a}{b}},
$$

e seja $V(a, h)=-V_{0}$, com

$$
V_{0}=h \frac{I}{\pi a^{2} g} .
$$

Suponhamos agora que aí, em $z=h$, fechássemos o cabo com um disco metálico de condutividade finita, (como comentado na Seção 1) perfazendo o espaço entre o condutor interno e o externo. O disco estaria então em $r=b$ a potencial zero e em $r=a$ ao potencial $-V_{0}$, como o fio central nesta seção. Fluiria então uma certa corrente convergente de $b$ para $a$, com a configuração de potencial como na Eq.7, já que a simetria é também cilíndrica (satisfazendo a parte radial da Eq.4). Ou seja, o potencial no ar do cabo co-axial em $z=h$ coincide com aquele no interior da tampa, pela conservação da componente tangencial do campo elétrico. $\mathrm{O}$ valor $I_{1}$ da corrente dependerá da condutividade e da espessura do disco empregado. Notemos que o condutor central e a tampa, ambos resistivos, formam um conjunto de resistências em paralelo.

No tratamento até aqui, o potencial em $z=0$ foi tomado como zero e o da seção em $z=h$, no fio central, como - $V_{0}$, Eq.8. Somando-se $V_{0}$ aos potenciais considerados, teremos a situação mostrada na Fig.2 para o cabo co-axial finito com corrente $I$. A pilha de força eletromotriz $V_{0} \mathrm{e}$ 
a ligação à terra no fio central em $z=h$ fixam os potenciais necessários.

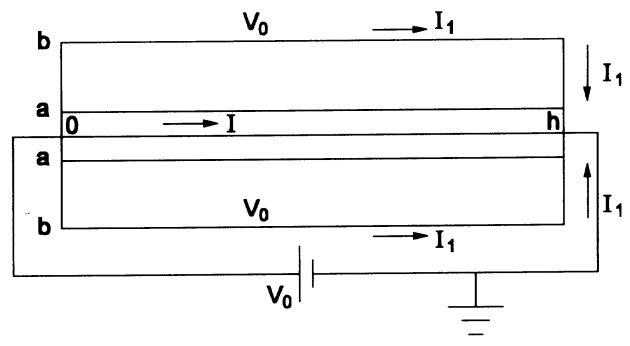

Figura 2. Cabo co-axial finito, fechado também em $z=h$ com placa circular de condutividade finita, ao longo da qual converge a corrente $I_{1}$, do cilindro exterior ao condutor central. $\mathrm{O}$ valor de $I_{1}$ depende da condutividade e da espessura do disco empregado. $\mathrm{O}$ potencial $V_{0}$ é aplicado ao condutor central (e ao cilindro) em $z=0$, e em $z=h$ está à terra. $\mathrm{O}$ sentido da corente $I$ está indicado.

\section{As cargas induzidas no cabo finito}

Consideremos agora o cabo finito como indicado no fim da seção anterior. Cargas superficiais estão induzidas na superfície do fio condutor interno, na superfície interna do condutor perfeito externo, nos discos circulares perfurados, em $z=0$ e em $z=h$. Elas são calculadas do campo elétrico $\vec{E}(r, z)$ que, da Eq.6, é dado por

$$
\vec{E}(r, z)=\frac{I}{\pi a^{2} g}\left(\frac{-z}{r \ln \frac{b}{a}} \hat{r}+\frac{\ln \frac{r}{b}}{\ln \frac{a}{b}} \hat{k}\right)
$$

em que $\hat{k}$ é o versor da direção $z$. Isto é, o campo radial é negativo e o axial é positivo. As densidades de carga na superfície do fio interno, $\sigma(a, z)$, no cilindro em $b, \sigma(b, z)$, e nos discos em $z=0$ e em $z=h$, respectivamente, $\sigma(r, 0)$ e $\sigma(r, h)$, são

$$
\begin{gathered}
\sigma(a, z)=\frac{-b}{a} \sigma(b, z)=\frac{\varepsilon_{0} I z}{\pi a^{3} g \ln \frac{a}{b}}, \\
\sigma(r, 0)=-\sigma(r, h)=\frac{\varepsilon_{0} I \ln \frac{r}{b}}{\pi a^{2} g \ln \frac{a}{b}}, \quad a<r<b,
\end{gathered}
$$

sendo $\varepsilon_{0}$ a permitividade do vácuo.

Integrando-se a densidade de carga nas superfícies, interna de raio $a$ e externa de raio $b$, vê-se que as cargas nessas duas superfícies são iguais e opostas, de módulo $Q_{1}$ dado por

$$
Q_{1}=\frac{\varepsilon_{0} h^{2} I}{a^{2} g \ln \frac{b}{a}}
$$

e negativa sobre a superfície do fio central. A distribuição resultante de cargas na superfície do fio está mostrada na Fig.3. Ela é de carga negativa e cresce do pólo positivo da pilha, à esquerda, ao pólo negativo, criando campo positivo no interior do fio. Uma carga positiva seria compelida a mover-se no sentido positivo de $z$, e uma negativa, saindo de $z=h$, mover-se-ia no sentido negativo. Este é o caso realista da condução metálica, em que os elétrons são as cargas móveis enquanto que os átomos que perderam sua carga de compensação - os íons - são as cargas fixas. Se o fio fosse material semicondutor, os dois casos de condução positiva (por buracos) e negativa (por elétrons) poderiam ocorrer, valendo a pena lembrar que mesmo na condução positiva, são os elétrons as cargas móveis. Voltando à Fig.3, cargas positivas estão depositadas na superfície interna do cilindro envolvente. Nos discos, não mostradas, são também induzidas cargas iguais e opostas, de módulo $Q_{2}$, contidas entre $a$ e $b$,

$$
Q_{2}=\frac{-\varepsilon_{0} I}{g}\left[1+\frac{b^{2}-a^{2}}{2 a^{2} \ln \frac{a}{b}}\right] .
$$

$Q_{1}$ deve ser, em geral, maior que $Q_{2}$, visto que $Q_{2}$ não depende de $h$. Ainda, como $\varepsilon_{0}=8,85 \times 10^{-12} \mathrm{~F} / \mathrm{m}$ e a condutividade do cobre, por exemplo, é $6,25 \times 10^{7}(\Omega \mathrm{m})^{-1}$, vê-se que, realmente, as cargas envolvidas no carregamento das partes metálicas são, em geral, muito pequenas, ou seja, que um tempo pequeno, da ordem de $\left(\frac{h}{a}\right)^{2} \cdot 10^{-19} \mathrm{~s}$, de circulação da corrente $I$ seria suficiente para suprí-las. Para o carvão, 2000 vezes mais resistivo, o tempo seria correspondentemente 2000 vezes mais longo. Esse tempo será da ordem de segundo para $g \approx 10^{-11} h^{2} / a^{2}(\Omega \mathrm{m})^{-1}$.

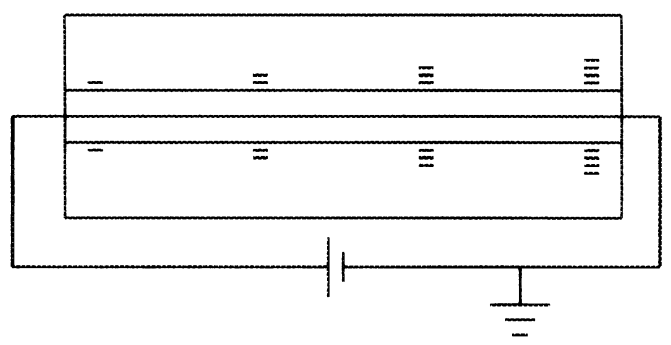

Figura 3. Distribuição esquemática da carga superficial para o cabo alimentado como na Fig.2. As cargas nas tampas não são mostradas.

O sinal negativo das cargas depositadas na superfície do fio e mostradas na Fig.3 resulta das ligações assumidas. Se as tampas em $z=0$ e $z=h$ fossem invertidas, mantendose os potenciais, a distribuição de cargas sobre o fio central seria positiva e decrescendo do pólo positivo ao negativo.

\section{Fios finos}

No nosso tratamento, o fio condutor é reto e necessita-se do cilindro exterior para fixação de condição de contorno adequada. Já a solução na simetria toroidal mencionada na Introdução [10], prescinde da presença desta referência de potencial, que naturalmente é o infinito. Para o caso em que o toróide é um fio fino e em pontos próximos a este, a diferença essencial entre a solução em [10] (fazendo-se aí a constante $A=0$ ) e a nossa Eq.6 vem do referenciamento do potencial, de tal forma que neste é o centro geométrico da configuração toroidal e na Eq.6 no cilindro externo. A 
densidade de carga na superfície do fio também varia linearmente ao longo dele, decrescendo do pólo positivo ao negativo, e trocando de sinal a meio caminho entre os pólos. A carga total fornecida pela pilha ao fio é nula (como no nosso caso). Do que foi dito, podemos dar uma expressão aproximada para a variação da carga $d q$ no comprimento $d s$ de fio, sendo $\bar{R}$ o raio médio do circuito:

$$
\frac{d q}{d s}=\frac{\varepsilon_{0}(\pi-s / \bar{R}) V_{0}}{\ln \bar{R} / a}
$$

sendo $s$ contado do pólo positivo ao potencial $V_{0}$.

Conclui-se também que nos fios e nas superfícies exteriores ao cabo, como nas Figs. 2 e 3, cargas (que não calculamos), devem estar aí depositadas.

\section{Considerações finais}

No presente artigo argumentou-se que a pilha, além de fornecer a força eletromotriz, provê as cargas superficiais, com densidade variando linearmente de forma a criar a configuração de campo uniforme no interior do fio condutor, necessária para o estabelecimento de corrente uniforme, o que é, conceitualmente, importante, ao fazer o vínculo entre a Eletrostática e a Eletrodinâmica. O fechamento do cabo, realizado na Seção 4, permite tratamento mais real- ista do problema, já que evita os infinitos de potencial e de extensão do cabo.

\section{Referências}

[1] J. D. Jackson, Am. J. Phys. 64, 855 (1996).

[2] A. Sommerfeld, Electrodynamics, Academic Press 1952, seção 17.

[3] M. Jouguet, Le Champ Électromagnétique, Librairie Armand Colin, 1948, seção 42.

[4] B. A. Sherwood e R. W. Chabay em http://www4.ncsu.edu:8030/rwchabay/mi/circuit.pdf

[5] O. Jefimenko, Phys. Teach. 15, 52 (1977).

[6] N. W. Preyer, Am. J. Phys. 68, 1002 (2000).

[7] A. K. T. Assis, W. A. Rodrigues Jr. e A. J. Mania, Found. Phys. 29, 729 (1999).

[8] M. A. Heald, Am. J. Phys. 52, 522 (1984).

[9] A. K. T. Assis e A. J. Mania, Rev. Bras. Ens. Fis. 30, 469 (1999).

[10] J. A. Hernandes e A. K. T. Assis em http://www.ifi.unicamp.br/ julioher/hernandes.pdf

[11] O. Jefimenko, Am. J. Phys. 30, 19 (1962). 